\title{
Aneurisma idiopático de la arteria pulmonar. Presentación de un caso y revisión de la literatura
}

\author{
M. E. DE TOMÁS LABAT, S. BELTRÁN BELTRÁN', S. MOLINA NAVEROS², \\ F. NAVARRO BOTELLA ${ }^{2}$, D. ÁLVAREZ SOTO ${ }^{2}$, E. PÉREZ MORO, G. ALDAMIZ- \\ ECHEVARRÍA CASTILLO
}

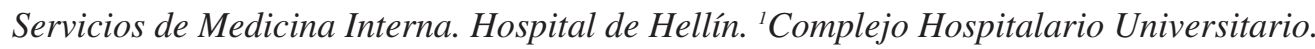
Albacete. ${ }^{2}$ Medicina Familiar y Comunitaria. Hospital de Hellín. ${ }^{3}$ Unidad de Cirugía Cardiaca. Clínica Recoletas. Albacete

IDIOPATHIC PULMONARY ARTERY ANEURYSM: REPORT A CASE AND REVIEW OF THE LITERATURE

\begin{abstract}
RESUMEN
Mujer de 63 años de edad con hipertensión arterial esencial, temblor esencial e insuficiencia venosa crónica en miembros inferiores; que acude a la Consulta Externa de Medicina Interna por su insuficiencia venosa crónica, objetivandose en la exploración física un soplo sistólico II/VI en borde esternal izquierdo y signos de insuficiencia venosa crónica en miembros inferiores. En la radiografía de tórax se encontró como hallazgo casual, una imagen vascular bien delimitada a nivel del mediastino superior izquierdo, motivo por el que se realizaron posteriormente diversas exploraciones complementarias progresivamente más agresivas, que incluyeron una angiografía pulmonar, que reveló la presencia de una dilatación aneurismática de las arterias pulmonares común e izquierda. Con estos hallazgos, la paciente fue sometida a intervención quirúrgica, realizándose resección parcial y plicatura de las arterias pulmonares común e izquierda hasta reducir a un diámetro de 2-3 centímetros, con un postoperatorio sin complicaciones y permaneciendo asintomática tras la cirugía.
\end{abstract}

PALABRAS CLAVE: Aneurisma de la arteria pulmonar. Aneurisma idiopático. Dilatación patológica de la arteria pulmonar.

\begin{abstract}
Woman of 63 years old with essential arterial hypertensión, essential trembling and chronic venous insufficiency of inferior members; she came to the Internal Medicine outpatients department because of her chronic venous insufficiency, objectifying in the physical examination a systolic heart murmur II/VI in the left sternal border and many signs of chronic venous insufficiency in the inferior members. In the chest radiograph was found as an accidental finding, a properly delimited vascular image in the left upper mediastino, for this reason there were later realized diverse complementary examinations gradually more aggressive, that included a pulmonary angiography which revealed the presence of an aneurysmatic dilatation in the common and left pulmonary arteries. With these findings, the patient was undergoing a surgical operation, in which was realized a partial resection and folder to the common and left pulmonary arteries to reduce diameter to 2-3 centimetres, with a postoperative period without complications and staying without symptoms after the surgery.
\end{abstract}

KEY WORDS: Pulmonary artery aneurysm. Idiopathic aneurysm. Pulmonary artery pathologic dilatation.

De Tomás Labat ME, Beltrán Beltrán S, Molina Naveros S, Navarro Botella F, Álvarez Soto D, Pérez Moro E, Aldamiz-Echevarría Castillo G. Aneurisma idiopático de la arteria pulmonar. Presentación de un caso y revisión de la literatura. An Med Interna (Madrid) 2005; 22: 329-331.

\section{INTRODUCCIÓN}

El aneurisma de la arteria pulmonar es la dilatación patológica del tronco de la arteria pulmonar y/o de una o ambas de sus ramas principales. Es una entidad clínica rara y de difícil diagnóstico por su baja prevalencia, porque suele presentarse con síntomas inespecíficos o incluso en pacientes asintomáticos, y porque puede pasar desapercibida por su evolución clínica o por confundirse radiológicamente con adenopatías u otro tipo de masas mediastínicas o pulmonares, resultando ser con cierta frecuencia un hallazgo autópsico (1-3).

Presentamos el caso de una paciente con un aneurisma idiopático de las arterias pulmonares común e izquierda, que cursó de forma asintomática y que fue un hallazgo casual en un control radiológico torácico.

\section{CASO APORTADO}

Mujer de 63 años de edad con hipertensión arterial esencial en tratamiento con valsartan ( $80 \mathrm{mg} /$ día), temblor esencial en tratamiento con propanolol ( $80 \mathrm{mg} / \mathrm{día})$, insuficiencia venosa crónica en miembros inferiores en tratamiento con medidas higiénico-posturales, y sin otros antecedentes de interés. Acude a la consulta externa de Medicina Interna para valoración y seguimiento del cuadro clínico de insuficiencia venosa crónica en miembros inferiores, sin referir ninguna otra sintomatología. La exploración física estaba dentro de 
la normalidad, salvo la presencia de soplo sistólico grado II/VI en borde esternal izquierdo en la auscultación cardiaca y signos de insuficiencia venosa crónica en miembros inferiores. En las exploraciones complementarias destacaba hemograma, VSG, coagulación, bioquímica y orina normales. ECG: normal. Radiografía de tórax: imagen vascular bien delimitada a nivel de mediastino superior izquierdo (Fig. 1). Ecocardiografía doppler: normal. TAC torácica con contraste intravenoso: dilatación aneurismática de la arteria pulmonar izquierda de 47x75 mm de diámetro (Fig. 2), sin otros hallazgos. Cateterismo cardiaco con angiografía pulmonar y coronaria: coronarias sin lesiones y dilatación aneurismática del tronco de la arteria pulmonar y de la arteria pulmonar izquierda. La paciente fue remitida al Servicio de Cirugía Cardiovascular de referencia, donde fue sometida a intervención quirúrgica, objetivándose en el acto quirúrgico una arteria pulmonar derecha de calibre normal y realizándose resección parcial y plicatura de las arterias pulmonares común e izquierda hasta reducir a un diámetro de 2-3 centímetros, cursando el postoperatorio sin complicaciones y permaneciendo asintomática tras la cirugía.

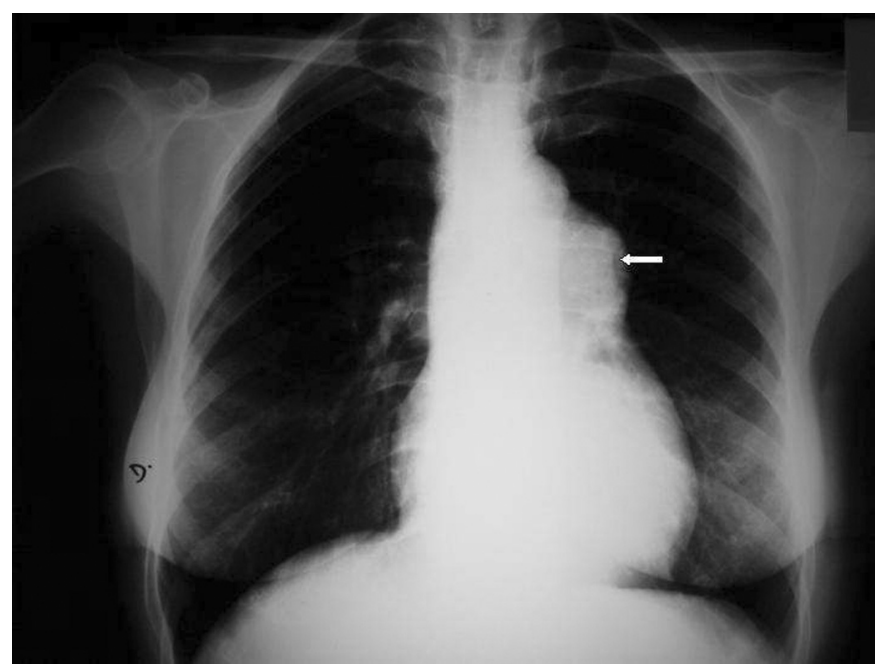

Fig. 1. Radiografía de tórax posteroanterior: imagen vascular bien delimitada a nivel de mediastino superior izquierdo (flecha).

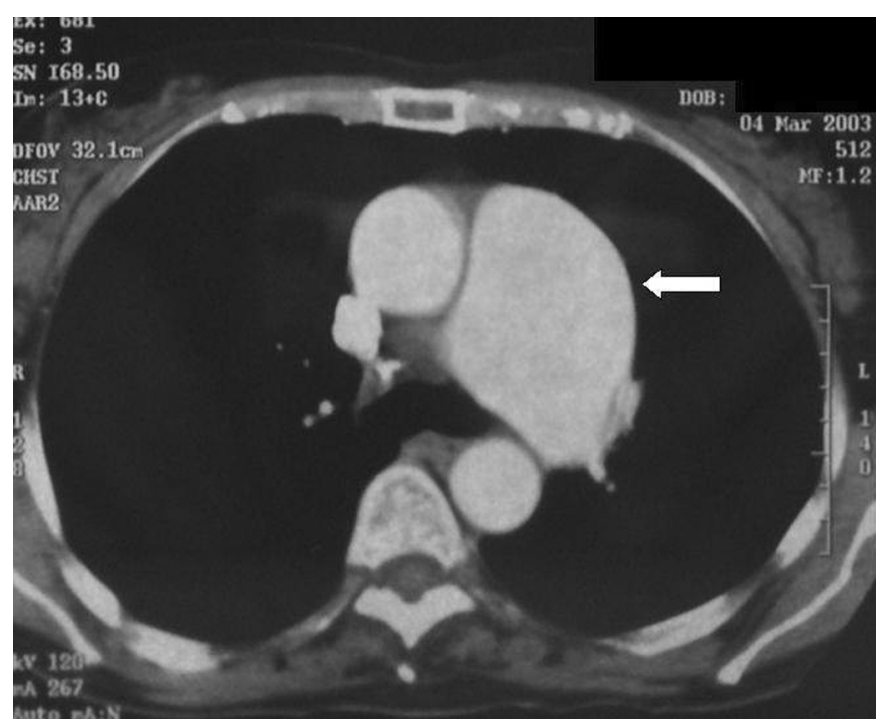

Fig. 2. TAC torácica con contraste intravenoso: dilatación aneurismática de la arteria pulmonar izquierda de $47 \times 75 \mathrm{~mm}$ de diámetro (flecha).

\section{DISCUSIÓN}

Los aneurismas de la arteria pulmonar (AAPs) se dividen en dos grupos: los que presentan comunicación arteriovenosa, que generalmente son congénitos y se asocian a defectos genéticos o a la telangiectasia hereditaria hemorrágica (enfermedad de Osler-Weber-Rendu), y los que no la presentan. En estos últimos hay una serie de factores predisponentes como infecciones, anormalidades cardiacas o vasculares estructurales, hipertensión pulmonar y traumas vasculares, existiendo en la mayoría de casos una interrelación entre varios de ellos $(2,3)$. Por tanto, en gran parte de los AAPs concurren una serie de situaciones fisiopatológicas previas al desarrollo del aneurisma: el aumento del flujo pulmonar, la existencia de una hipertensión pulmonar y/o la coexistencia de una patología que afecta a la pared vascular. En los casos infrecuentes en que el AAP no es debido a ninguna anomalía funcional ni estructural del sistema cardiovascular, recibe el nombre de AAP idiopático $(1,4,7)$. Nuestro caso correspondería a dicha entidad clínica, ya que se excluyeron las causas y los factores predisponentes para el desarrollo de un AAP.

Las manifestaciones clínicas cuando existen, ya que hay casos que cursan totalmente asintomáticos y son hallazgos casuales de anormalidades vasculares torácicas en estudios radiológicos realizados por otros motivos, suelen ser síntomas inespecíficos como disnea, hemoptisis, tos, dolor torácico o palpitaciones. También puede cursar, aunque es infrecuente, con insuficiencia cardiaca derecha y soplo sistólico, diastólico, a veces con frémito, audibles en el segundo y tercer espacios intercostales izquierdos $(2,4)$. La hemoptisis, generalmente, indica inestabilidad del aneurisma y es un indicador de la necesidad de intervención terapeútica $(2,3)$. La angiografía pulmonar se ha considerado la prueba de elección para el diagnóstico definitivo del AAP, así como una técnica terapéutica en determinados casos. Sin embargo según algunos autores su realización sistemática debería reservarse como prueba diagnóstica previa al tratamiento quirúrgico, ya que no aporta datos más precisos en los aneurismas de evolución presumiblemente benigna (1), y porque actualmente la tomografía computerizada y la resonancia magnética son alternativas diagnósticas que permiten confirmar el diagnóstico de AAP de forma incruenta $(1,2,5)$. La ecocardiografía con registro doppler puede, en algunos casos, llevar al diagnóstico y permite descartar anormalidades cardiacas estructurales asociadas $(1,5)$. La radiología simple torácica sólo permite la sospecha diagnóstica, al detectarse anormalidades vasculares torácicas o signos indirectos como hipertrofia o dilatación del ventrículo derecho y silueta cardiaca triangular (silueta de Laubry y Bordet) debido a la rotación del corazón por la dilatación de la arteria pulmonar (2,3). El pronóstico de los AAPs no está claro y es variable, ya que su historia natural es difícil de determinar $(2,6)$, lo que provoca una disyuntiva terapéutica que impide un criterio uniforme a la hora de elegir entre un tratamiento quirúrgico precoz o la vigilancia periódica (1). El pronóstico del AAP idiopático se considera benigno $(1,4)$.

El tratamiento del AAP puede ser médico o quirúrgico. El tratamiento médico es limitado y consiste en la vigilancia periódica, el control de la hipertensión pulmonar y de los factores predisponentes, y esta reservado para aneurismas de evolución benigna $(1,3,7)$. El tratamiento quirúrgico esta indicado en los aneurismas inestables, con hemoptisis, y en los que se describe la posibilidad de una evolución 
fatal por alto riesgo de rotura y mayor mortalidad: los aneurismas micóticos y los asociados a enfermedad de Behcet, enfermedad de Erdheim, síndrome de Hughes-Stovin, síndrome de Marfan, síndrome antifosfolípido primario y telangiectasia hereditaria hemorrágica $(1,3,8,13,14)$. Sin embargo, recientemente, se han descrito casos de AAPs asociados a la enfermedad de Behcet y al síndrome de Hughes-Stovin que han regresado tras tratamiento inmunosupresor (10-12). En nuestro caso, aunque la paciente estaba asintomática, el tamaño del aneurisma con el consiguiente riesgo de rotura, fue lo que determinó la indicación del tratamiento quirúrgico. Según la localización, el número y la etiología se optará por una técnica quirúrgica u otra. En los AAPs que afectan al tronco o a sus ramas principales, las posibilidades terapeúticas son la aneurismectomía o aneurismorrafia. Cuando son más periféricos, la embolización es la técnica de elección, ya que es una técnica con mínima morbilidad, aunque se puede emplear también la resección, y cuando no es posible otra técnica, la lobectomia de los más periféricos $(1,3,9,16-18)$.

\section{Bibliografía}

1. Palma JC, Sciaccaluga C, Antón J, Ramos VM. Aneurisma de la arteria pulmonar. Presentación de un caso. Rev Esp Cardiol 1999; 52: 148-150.

2. Chung CW, Doherty JV, Kotler R, Finkelstein A, Dresdale A. Pulmonary artery aneurysm presenting as a lung mass. Chest 1995; 108: 11641166.

3. Bartter T, Irwin RS, Nash G. Aneurysms of the pulmonary arteries. Chest 1988; 94: 1065-1075.

4. Canela P, Vergara A, Otero E, González V. Dilatación idiopática de la arteria pulmonar. Presentación de un caso. Rev Clin Esp 1985; 176: 158-159.

5. Shah HR, Stark JE, Buckner CB. Magnetic resonance imaging of pulmonary artery aneurysm. South Med J 1991; 84: 1026-1028.

6. Arom KU, Richardson JD, Grover FL. Pulmonary artery aneurysm. Am Surg 1978; 44: 688-692.

7. Van Res MT, Westermann CJ, Postmus PE, Schramel FM. Untreated idiopathic aneurysm of the pulmonary artery: long-term follow up. Respir Med 2000; 94: 404-405.

8. Chung MH, Lee HG, Kwon SS, Kim YS, Park SH. Pulmonary arterial aneurysms in primary antiphospholipid antibody syndrome. J Comput Assist Tomogr 2002; 26: 608-612.

9. Bozkurt AK. Embolisation in Behcet's disease. Thorax 2002; 57: 469470 .

10. Aktogu S, Erer OF, Urpek G, Soy O, Tibet G. Multiple pulmonary arte-

rial aneurysms in Behcet's disease: clinical and radiologic remission after cyclophosphamide and corticosteroid therapy. Respiration 2002; 69: 178-181.

11. Ali-Munive A, Varon H, Maldonado D, Torres-Duque C. Aneurisma gigante de la arteria pulmonar y trombosis venosa periférica (síndrome de Hughes-Stovin): regresión con terapia inmunosupresora. Arch Bronconeumol 2001; 37: 508-510.

12. Acican T, Gurkan OU. Azathiopine-steroid combination therapy for pulmonary arterial aneurysms in Behcet's disease. Rheumatol Int 2001; 20: $171-174$.

13. Koh KK, Kim SS, Park CS, Lee SC, Oh BH. Pulmonary artery aneurysm associated with hereditary hemorrhagic telangiectasia. Int J Cardiol 1994; 45: 227-229.

14. Lertsapcharoen $\mathrm{P}$, Chottivittayatarakorn $\mathrm{P}$, Benjacholamas V. Mycotic aneurysms of the pulmonary arteries. Heart 2002; 88: 524.

15. Telli HH, Gormus N, Temizhan A, Solck H. Giant pulmonary artery aneurysm. Eur J Cardiothorac Surg 2002; 21: 928.

16. Mann P, Seriki D, Dodds PA. Embolism of an idiopathic pulmonary artery aneurysm. Heart 2002; 87: 135.

17. Nair KK, Cobanoglu AM. Idiopathic main pulmonary artery aneurysm. Ann Thorac Surg 2001; 71: 1688-1690.

18. Kuwaki K, Morishita K, Sato H, Urita R, Abe T. Surgical repair of the pulmonary trunk aneurysm. Eur J Cardiothorac Surg 2000; 18: 535-539. 